\title{
Wave-driven dynamo action in spherical MHD systems
}

\author{
K. Reuter, ${ }^{1}$ F. Jenko, ${ }^{1}$ A. Tilgner, ${ }^{2}$ and C. B. Forest $^{3}$ \\ ${ }^{1}$ Max-Planck-Institut für Plasmaphysik, EURATOM Association, \\ Boltzmannstraße 2, D-85748 Garching, Germany \\ ${ }^{2}$ Institute of Geophysics, University of Göttingen, \\ Friedrich-Hund-Platz 1, 37077 Göttingen, Germany \\ ${ }^{3}$ Department of Physics, University of Wisconsin-Madison, \\ 1150 University Avenue, Madison, Wisconsin 53706, USA
}

(Dated: October 30, 2018)

\begin{abstract}
Hydrodynamic and magnetohydrodynamic numerical studies of a mechanically forced two-vortex flow inside a sphere are reported. The simulations are performed in the intermediate regime between the laminar flow and developed turbulence where a hydrodynamic instability is found to generate internal waves with a characteristic $m=2$ zonal wave number. It is shown that this time-periodic flow acts as a dynamo although snapshots of the flow as well as the mean flow are not dynamos. The magnetic fields' growth rate exhibits resonance effects depending on the wave frequency. Furthermore, a cyclic self-killing and self-recovering dynamo based on the relative alignment of the velocity and magnetic fields is presented. The phenomena are explained in terms of a mixing of non-orthogonal eigenstates of the time dependent linear operator of the magnetic induction equation. The potential relevance of this mechanism to dynamo experiments is discussed.
\end{abstract}

PACS numbers: 91.25.Cw, 47.65.-d, 47.20.-k

\section{INTRODUCTION}

It is well-established theoretically that magnetic fields which emanate from the Earth and from celestial bodies are generated by flows of electrically conducting fluids in their interiors, a mechanism which is commonly referred to as the magnetohydrodynamic (MHD) dynamo effect [1, 2]. In recent years, significant effort was devoted to the experimental verification of dynamo theory in simply connected spherical [3, 4, 5] and cylindrical [6, 7] impeller-driven flows of liquid sodium. While the latter setup has ultimately demonstrated a self-excited dynamo, it has turned out that turbulence strongly inhibits the dynamo process in both experiments. A review on dynamo experiments is given in Ref. [8]. Detrimental effects of turbulence on the excitation threshold of large-scale dynamos were confirmed by numerical simulations of MHD systems with well-defined mean-flows in periodic-box geometry [9, 10, 11, 12] as well as in spherically bounded geometry [13, 14, 15].

In the present paper, we study numerically the regime between turbulence and the laminar state of an electrically conducting two-vortex flow inside a sphere which is similar to the flow realized in the Madison Dynamo Experiment 3, 4, 5]. The structure of the manuscript is as follows. The numerical model is presented in section [II Section [II discusses purely hydrodynamic simulations which are performed in the sub-turbulent regime. It is found that a hydrodynamic instability leads to traveling internal waves, their dominant component being symmetric under rotations by $\pi$. In section [IV] we turn to MHD investigations of the flows introduced in the previous section. It is found that the presence of the waves which correspond to smoothly oscillating large-scale fluctuations supports the dynamo instability. Artificially changing the wave's frequency yields a resonance effect, i.e. the dynamo is found to operate most efficiently at certain frequencies whereas dynamo action ceases for frequencies which are too low or too high. Moreover, a nonlinear dynamo is reported which undergoes a cycle of self-killing and self-recovering events. We find that this behavior is related to phase shifts between characteristic magnetic and hydrodynamic oscillations which are imposed during the transitions. These phase shifts translate to changes in the relative alignment of the velocity and magnetic fields. Section $\nabla$ concludes with a discussion and interpretation of the numerically obtained results. We interpret the MHD phenomena in the framework of a dynamo mechanism based on the mixing of non-orthogonal eigenstates of the time-dependent linear operator of the induction equation [16], thus confirming its relevance to a self-consistent dynamo model.

\section{NUMERICAL MODEL}

The magnetic (B) and velocity (v) fields which describe an incompressible electrically conducting fluid are governed by the induction equation (11) and the NavierStokes equation (21),

$$
\begin{aligned}
\frac{\partial \mathbf{B}}{\partial t} & =\nabla \times(\mathbf{v} \times \mathbf{B})+\lambda \nabla^{2} \mathbf{B}, \\
\frac{\partial \mathbf{v}}{\partial t}+(\mathbf{v} \cdot \nabla) \mathbf{v} & =-\nabla p+\nu \nabla^{2} \mathbf{v}+\mathbf{j} \times \mathbf{B}+\mathbf{F},
\end{aligned}
$$

along with the constraints $\nabla \cdot \mathbf{B}=\nabla \cdot \mathbf{v}=0$. Here, $\lambda$ is the magnetic diffusivity, $p$ is the pressure, $\mathbf{j}=\mu_{0}^{-1} \nabla \times \mathbf{B}$ is the current density, $\nu$ is the viscosity, and $\mathbf{F}$ is a forcing term. A constant mass density $\rho=1$ is assumed. In nondimensional form, the problem is characterized by two control 
parameters, the Reynolds number $\operatorname{Re}=L V \nu^{-1}$ and the magnetic Reynolds number $\mathrm{Rm}=L V \lambda^{-1}$, where $L$ and $V$ denote length and velocity scales which are characteristic of the system under consideration. We choose $L=1$ and $V=\overline{v_{\text {rms }}}$, where $v_{\text {rms }}=\sqrt{\left\langle\mathbf{v}^{2}\right\rangle}$ is the spatial rms velocity. Here, the angle brackets denote averaging in space, and the overline denotes averaging in time which is performed during the quasi-stationary phase of the flow after all initial bifurcations. The characteristic timescale of the flow is given by the eddy turnover time $\tau_{\nu}=L / V$, whereas the magnetic diffusion time $\tau_{\sigma}=\operatorname{Rm} \tau_{\nu}$ is the timescale relevant to the magnetic field.

We solve numerically the MHD equations (11)-(2) in a sphere, using the parallel version of the DYNAMO code [13, 17]. It employs the standard pseudo-spectral method based on a poloidal-toroidal decomposition of the vector fields in combination with spherical harmonic expansions. Outer boundary conditions are the potential field solution for the magnetic field, and, unless stated otherwise, the zero-slip condition for the velocity field. The forcing is designed to produce an $s 2 t 2$ type of flow [18], which consists of two in the toroidal direction counter-rotating hemispherical cells with poloidal circulation in each cell, directed outwards to the poles and inwards in the equatorial plane. It is given by an axisymmetric localized body force $\mathbf{F}$ kept constant in time, which reads in a cylindrical coordinate system $(s, \phi, z)$

$$
\begin{aligned}
& F_{s}=0 \\
& F_{\phi}=\epsilon \operatorname{sgn}(z) s^{3} r_{d}^{-3} \sin \frac{\pi s}{2 r_{d}}+\gamma, \\
& F_{z}=(1-\epsilon) \operatorname{sgn}(z) \sin \frac{\pi s}{r_{d}}+\delta .
\end{aligned}
$$

Assuming a sphere of radius $r=1$, the driving is applied within $0.25<|z|<0.55, s<r_{d}=0.29$, with the parameters kept constant at $\epsilon=0.1, \gamma=0.05, \delta=0.3$. For sufficiently large $\mathrm{Rm}$, the resulting flow is a dynamo, the $(\ell=m=1)$ magnetic eigenmode growing fastest. The generation of this transverse dipole can be understood in the axisymmetric flow using a simple frozen-flux picture based on stretching, twisting, and folding of magnetic field lines [19].

In all simulations, the spherical harmonic expansions are triangularly truncated at degree and order $\ell_{\max }=$ $m_{\max }=20$, while 160 uniformly spaced radial points are used. To verify the simulation results obtained at the aforementioned resolution we have repeated a nonlinear run (the computation of flow $\mathrm{C}$ which is introduced in the following section) using $\ell_{\max }=m_{\max }=30$ and 320 gridpoints in radial direction. The bifurcations towards flow $\mathrm{C}$ as well as the growthrate of the magnetic field in the MHD case were fully reproduced which indicates that converged solutions are obtained already at the coarser resolution. Moreover, power spectra of the velocity fields in terms of $\ell$ show a drop-off by three to four orders of magnitude between the dominant and the highest wavenumber. The magnetic field is resolved similarly well. Relevant spectra are shown and discussed in

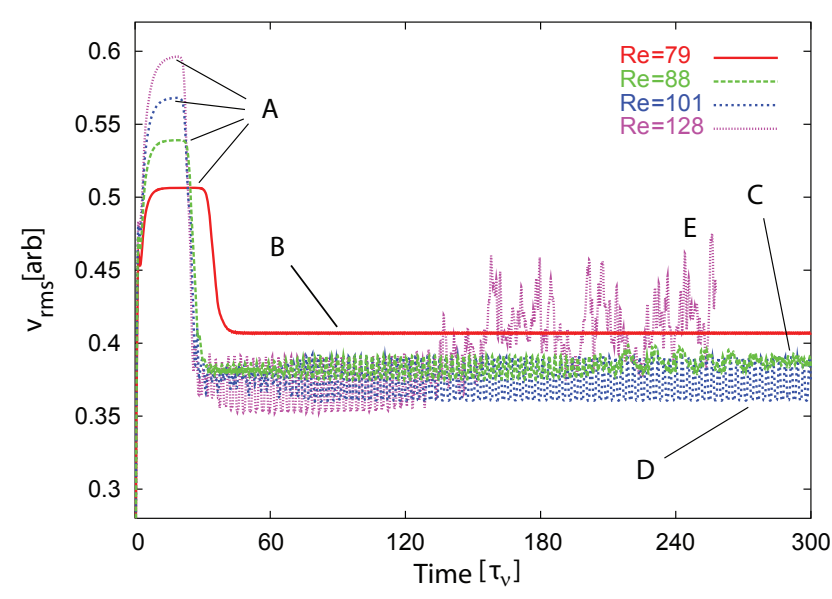

FIG. 1: Time traces of $v_{\text {rms }}$ for four $s 2 t 2$ flows driven by the body force $\mathbf{F}$, at Re being moderately above the stable region $\operatorname{Re}<65$, illustrating bifurcation sequences.

the following sections, cf. Figs. 4 and 12 After having introduced the numerical setup we now turn to an investigation of the hydrodynamics of the flow before we consider full MHD cases.

\section{HYDRODYNAMIC STUDIES}

We first investigate the dynamics of the unmagnetized flow, i.e. Eq. (2) is integrated forward in time with $\mathbf{B}=\mathbf{0}$. Starting from a fluid at rest, momentum is injected by the body force $\mathbf{F}$, and the time integration is performed until a statistically stationary state is reached. This procedure is repeated to scan over various decreasing viscosities, thus increasing Re. For Re $<65$, the flow reaches a stationary axisymmetric $(m=0)$ state. Above this threshold, the flow becomes hydrodynamically unstable. To exemplify subsequent temporal transitions, Fig. 1] shows time traces of $v_{\text {rms }}$ at $\operatorname{Re}=79,88,101,128$, the quasi-stationary states being labeled B, C, D, E. In each case, the flow first reaches an axisymmetric configuration which is characterized by an initial plateau (A). Simultaneously, non-axisymmetric modes with even $\ell$ and zonal wave number $m=2$ grow fastest at an exponential rate. Shortly before saturation occurs, the symmetry breaking becomes visible in physical space near the polar regions of the sphere as indicated in Fig.2(a). The previously circular cross sections of the jets towards the poles are stretched elliptically as it is depicted in Fig. 2 (b-d) for the flow D. The nonlinear saturation of this instability manifests itself in a relaxation of $v_{\text {rms }}$, cf. Fig. 1 . In physical space, traveling waves with $m=2$ symmetry emerge, their spatial structure being illustrated in Fig. 3. The wave propagation is directed opposite to the sense of rotation of the mean-flow in the individual hemisphere as prescribed by the driving, with the wave vector pointing in the zonal direction. With increasing 


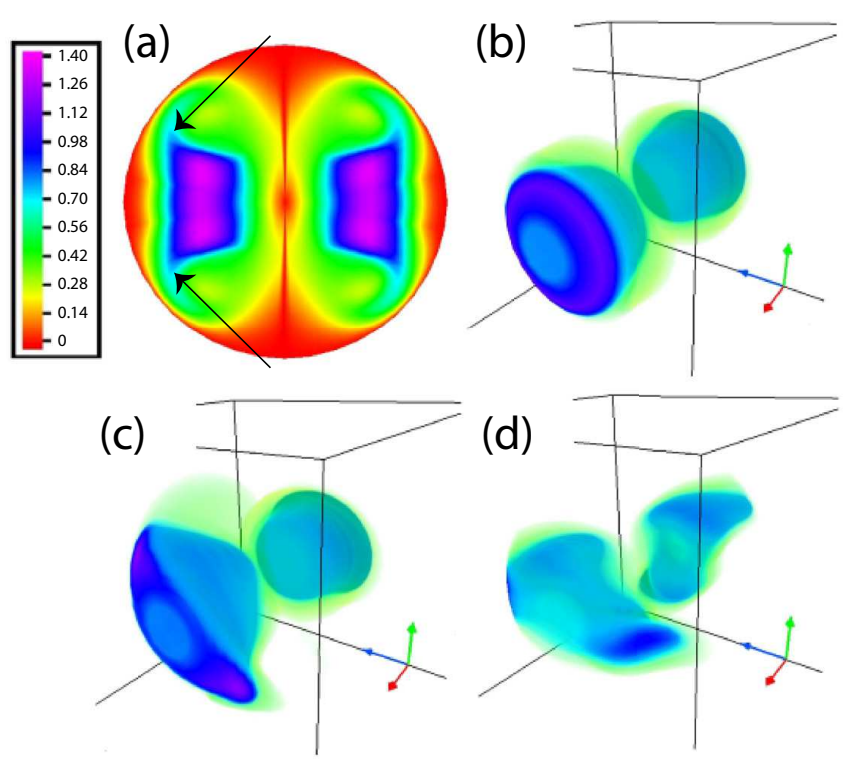

FIG. 2: Illustrations of the symmetry breaking in the flow towards state D. (a) Poloidal cross section of the energy density $\propto \mathbf{v}^{2}$ during the axisymmetric phase A. Arrows indicate the region where elliptical stretching occurs. (b-d) Time series depicting the spatial structure of large amplitudes of $\mathbf{v}^{2}$ inside the sphere $r<0.75$ as the instability grows.

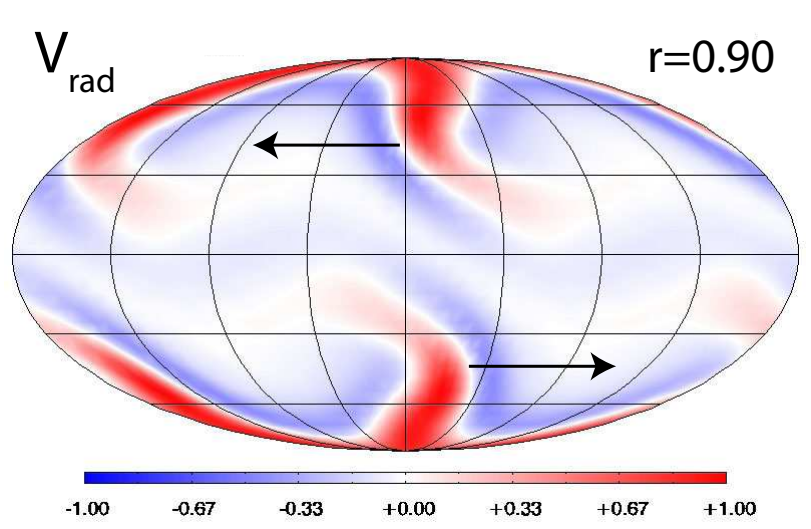

FIG. 3: Mollweide projection of the radial component of a velocity field snapshot at $90 \%$ of the sphere's radius during the statistically stationary state D. Arrows indicate the direction of wave propagation.

Re, the latitudinal extent of the wave grows towards the equator, coupling the two hemispheres and causing oscillatory fluid motions (cf. Fig. 1, states $\mathrm{C}$ and D compared to state B). As a result, the wave frequency decreases with increasing Re. With $\mathrm{Rm} \approx 44$ (see below), the frequencies in the lab frame turn out to be fast on the resistive timescale, namely $f_{\mathrm{C}} \approx 19.08 \tau_{\sigma}^{-1}=0.43 \tau_{\nu}^{-1}$ and $f_{\mathrm{D}} \approx 16.71 \tau_{\sigma}^{-1}=0.39 \tau_{\nu}^{-1}$. For $\operatorname{Re}>125$, turbulence develops, and the $m=2$ wave feature loses its clear structure (state E).

Fig. 4 displays time averaged energy spectra of the flows $\mathrm{C}$ and $\mathrm{D}$ in terms of spherical harmonic degree $\ell$
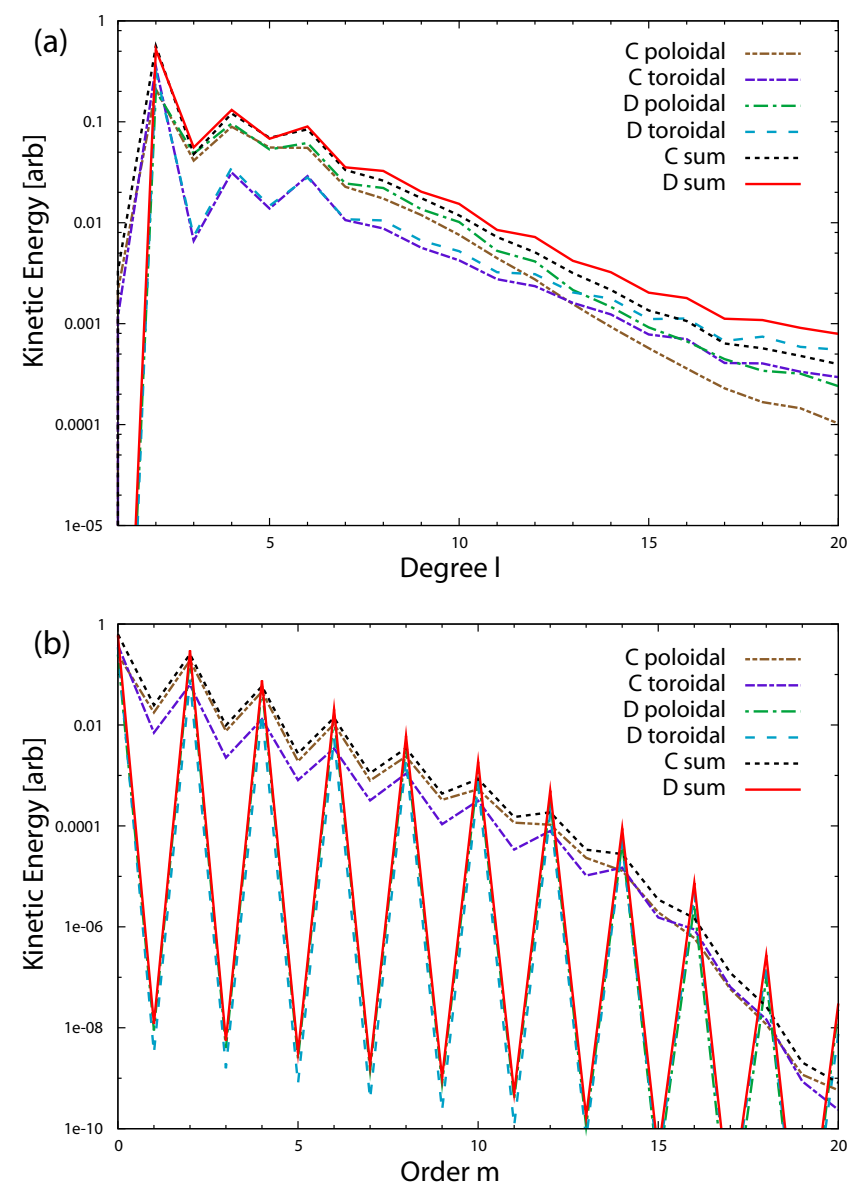

FIG. 4: Time-averaged spectra of the kinetic energy density in the flows $\mathrm{C}$ and $\mathrm{D}$ as functions of spherical harmonic degree $\ell$ (a) and order $m$ (b).

and order $m$. The time averaging was performed for several hundred $\tau_{\nu}$ to assure convergence. The $\ell$-spectra in Fig. 4 (a) peak at $\ell=2$ for both flows which is due to the imposed forcing. The spectra are overall similar except for the strength of the $l=1$ modes which refer to global circulation, however, these modes are dynamically not important in flow $\mathrm{C}$ and even less in $\mathrm{D}$ due to their small amplitudes. The spectra of flow C drop off somewhat faster than those of flow D which is to be expected due to the larger value of the kinematic viscosity in run C. The spectra in terms of the order $m$ shown in Fig. 目(b), however, yield differences between C and $\mathrm{D}$ concerning the amplitude of the modes with odd wavenumbers. In both cases, the spectra peak at $m=0$ which is due to the axisymmetric background flow. The dominant peak connected with non-axisymmetric modes is found at $m=2$ and caused by the wave feature, followed by peaks at higher harmonics with even $m$. The origin of the preference for even modes in flow D remains unclear. During the initial bifurcation sequence, the amplitudes of the odd $m$-modes drop off suddenly around $\tau_{\nu} \approx 70$ after a phase spectrally similar to $\mathrm{C}$ before the statistically stationary state $\mathrm{D}(\mathrm{Re}=101)$ is reached. 
The corresponding change of the flow becomes apparent in Fig. 1] as a change in the oscillatory behaviour of the respective time trace. Conversely, the flow $\mathrm{C}$ at $\mathrm{Re}=88$ starts off with odd $m$-modes at small amplitude after the initial transition $\mathrm{A}$. The respective time trace in Fig. 1 shows regular oscillations until, at $\tau_{\nu} \approx 210$, odd $m$-modes have grown sufficiently strong to become dynamically important. The change in the flow towards the statistically stationary state $\mathrm{C}$ manifests itself in a more irregular time trace of $v_{\text {rms }}$. Flows with metastable states switching between the characteristics of flow $\mathrm{C}$ and D were not observed, although this possibility may exist. It should be noted that, when going to higher Re, the $m$-spectra tend to be smoothed. A local peak at $m=2$ is still found, and can be interpreted as the signature of the most unstable modes excited by the hydrodynamic instability.

In the case of flow D, the temporal evolution of the dominant components of the spectrum is shown in the upper panel of Fig. 9. The time trace of the $(\ell=2, m=0)$ axisymmetric background flow [which will be abbreviated $(2,0)$ in the following] is modulated sinusoidally at frequency $f_{D}$ via nonlinear interactions with other modes. The $m=2$ wave feature corresponds to modes oscillating at a time-periodic non-sinusoidal pattern at an amplitude which is about $10 \%$ of the amplitude of the $(2,0)$ background flow. In the case of flow $\mathrm{C}$, the picture is overall similar, although the time traces show different characteristics concerning their periodicity and shape.

Additional numerical experiments were performed to better understand the origin of the wave phenomenon. Outer shear-layers which exist due to the zero-slip condition on the velocity field prove to be crucial for the wave generation. This boundary condition requires the three vector components of the velocity field to be zero at the outer boundary. It is intended to be an approximation of the experimental reality where liquid sodium is confined by a solid wall. Applying the stress-free boundary condition instead in a numerical experiment while leaving the forcing $\mathbf{F}$ unchanged fundamentally alters the nature of the hydrodynamic instability. The stress free boundary condition requires the radial component of the velocity field to be zero at the boundary, whereas the angular components evolve under the constraint of zero stress. As a result, a flow pattern with a strong zonal flow next to the boundary develops in each hemisphere. Stationary states with waves comparable to the flows C and D are not observed. These findings support the potential relevance of the results presented in this paper to existing dynamo experiments where boundary layers with strong shear exist in the flows next to the outer walls.

Moreover, the fact that $s 2 t 2$ flows were studied in the past by several groups, each of them using slightly different forcing functions or prescribed flows, motivates us to investigate the role of the forcing term on the wave generation. Applying the zero-slip condition, wavy states similar to C and D are found in the sub-turbulent regime when the flow is driven by the localized body force given in [13, 14], and, similarly, when the global $s 2 t 2$ profiles discussed in [18] are employed to drive the flow. In addition to the axisymmetric component, non-axisymmetric $m=2$ modes are found to dominate in these flows, however, modes with $m=1$ and $m=3$ wave numbers are dynamically important as well. A main difference between the three forcing schemes consists in the distance between the active region where momentum is injected, and the outer boundary. This distance is largest in our impeller model, Eq. 3, and is chosen to be smaller in the latter forcing schemes. The decrease affects the thickness of the boundary layer and thereby the rate of strain which in turn may govern which zonal mode $m$ is most unstable. A final comment on the influence of the forcing is made on the structure of the magnetic field in case of a dynamo. It turns out that a flow forced by Eq. 3 favors an $m=1$ dipole mode in the laminar case, in the intermediate sub-turbulent range on which we focus in the present paper, as well as in the presence of developed turbulence. An $m=0$ axial dipole as it was reported in [13, 14] has not been found using Eq. 3. We have, however, numerically confirmed its existence in turbulent flows when the forcing reported in [13, 14] is applied.

A more detailed investigation of the hydrodynamics of the $s 2 t 2$ flow is certainly required to shed light on the plausible conjecture that the previously discussed hydrodynamic instability and wave formation are universal features of the spherically bounded $s 2 t 2$ flow. The corresponding study, however, goes beyond the scope of the present manuscript. We now proceed towards the actual goal of the paper, namely the investigation of the dynamo properties of the sub-turbulent flows $\mathrm{C}$ and $\mathrm{D}$.

\section{MHD INVESTIGATIONS}

The $s 2 t 2$ class of dynamos has been the focus of several numerical investigations, pioneered by the work of Dudley and James [18] who solved the induction equation for a stationary prescribed two-vortex flow. Later-mainly concentrating on the role of turbulence on the dynamo process - fully time dependent flows in nonlinear MHD simulations were studied [13, 14, 15]. Until now, however, the effect of smoothly fluctuating fluid motions below the transition to developed turbulence has not been considered explicitly. This motivates us to investigate the temporal evolution of seed magnetic fields in the flows $\mathrm{C}$ and D. To this end, the magnetic field $\mathbf{B}$ is initialized with pseudo-random noise at small amplitude, and the induction equation is solved numerically using four different types of velocity fields $\mathbf{v}$ based on $\mathrm{C}$ and $\mathrm{D}$ : (i) time dependent flows $\mathbf{v}(\mathbf{x}, t)$, i.e. the coupled Eqs. (11)-(2) are integrated; (ii) snapshots $\mathbf{v}\left(\mathbf{x}, t_{i}\right), i=1 \ldots n$ of the flows kept fixed in time; (iii) time averaged velocity fields $\overline{\mathbf{v}}=\frac{1}{T} \int_{t_{0}}^{t_{0}+T} \mathbf{v}(\mathbf{x}, t) d t$, with $T>10^{2} \tau_{\nu}$; (iv) consecutive snapshots of the flows iterated at variable rates which allows to examine the effect of the wave frequency on the magnetic field's growth rate. Practically, the runs of type 


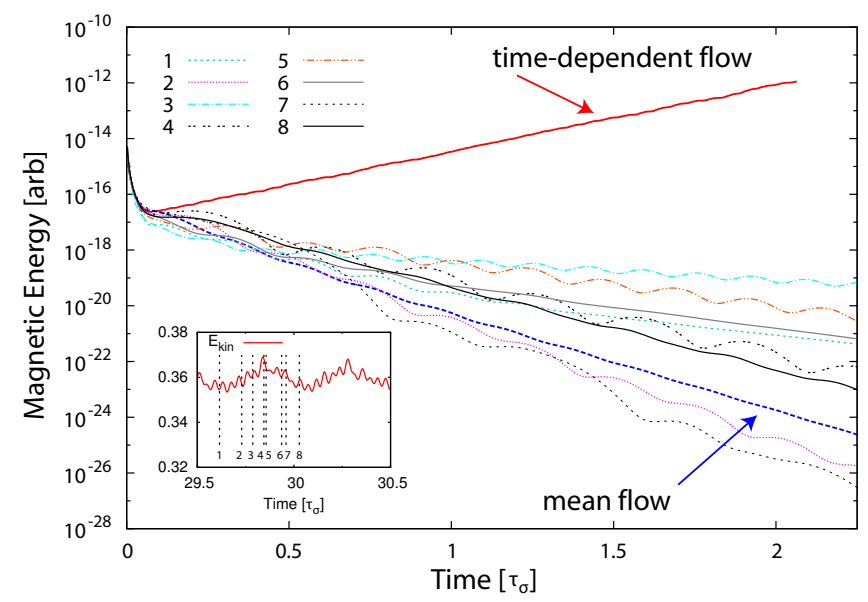

FIG. 5: Energies of the magnetic field over time, from simulations (i)-(iii) based on the flow $\mathrm{C}(\mathrm{Re}=88, \mathrm{Rm}=44)$.

(iv) are performed by subsequently reading previously saved snapshots from hard disk, while solving Eq. (1). Unless stated otherwise, all MHD runs discussed in the following are performed at $\mathrm{Rm} \approx 44$.

We first consider the flow C for which Fig. 5 summarizes the results of the numerical experiments (i)-(iii). Eight randomly selected snapshots - as indicated by the inset-show decaying magnetic fields. Moreover, the mean-flow is not a dynamo. Only the time dependent flow (i) shows a growing magnetic field solution. Clearly, the presence of time dependence in the velocity field in the form of periodic wave motion causes the magnetic field to grow. In fact, in the regime around $\mathrm{Re} \approx 100$, the dynamo threshold of the mean flow is $\operatorname{Rm}_{\mathrm{c}}(\overline{\mathbf{v}}) \approx 55$, which is significantly larger than the threshold of the time dependent flows, being $\mathrm{Rm}_{\mathrm{c}} \approx 32$. Fig. 6 displays the growth rate as a function of the relative wave frequency $f / f_{\mathrm{C}}$ obtained by performing experiment (iv). For comparison, the growth rate calculated from the selfconsistent run (i) is included. A window with positive growth rates $\left(f / f_{\mathrm{C}} \approx 0.55 \ldots 6.0\right)$ exists which contains two local maxima at $f / f_{\mathrm{C}} \approx 0.9$ and $f / f_{\mathrm{C}} \approx 4.4$ being symptomatic of resonant behavior. The first maximum happens to be in good agreement with the frequency of the hydrodynamic waves in run (i). Negative growth rates occur when the wave is either too fast or too slow. The latter finding reflects the fact that snapshots of the flow are not dynamos. Iterating through the velocity field snapshots in reversed order, i.e. inverting the direction of wave propagation without changing the shape of the waves, causes seed magnetic fields to decay in all investigated cases in the range $f / f_{\mathrm{C}}=-10.0 \ldots-0.1$. The observation of magnetic field amplification in a spherically bounded MHD dynamo model induced by hydrodynamic waves represents the key result of our work. A power spectrum of the growing magnetic field obtained from the nonlinear MHD simulation of flow $\mathrm{C}$ is given in Fig. 12, The magnetic field is clearly dominated by

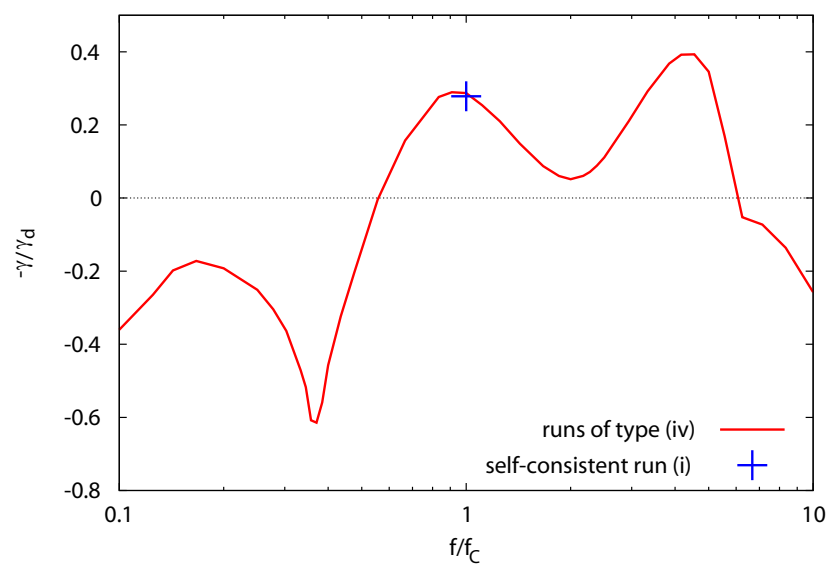

FIG. 6: Growth rate of the magnetic field, normalized by the negative free dipole decay rate, as a function of the relative wave frequency $f / f_{\mathrm{C}}$. The simulations were performed based on flow $\mathrm{C}$ at $\mathrm{Rm}=44$.

the $(1,1)$ dipole mode. The dynamo based on experiment (i) saturates to a stationary state without any time dependence in the velocity and magnetic fields. The kinetic and magnetic energies are roughly in equipartition. Before we turn towards a discussion of the physics governing the magnetic field growth in flow $\mathrm{C}$, we perform an analogous investigation of the flow D.

An intriguing phenomenon is found when the evolution of magnetic fields in flow D is studied. Performing run (i), a seed magnetic field first grows exponentially at a normalized rate $\gamma_{\mathrm{D}} \approx 0.46$, as it is indicated by the time trace of the magnetic energy in Fig. 7 Nonlinear feedback via the Lorentz force acts starting from $\tau_{\sigma} \approx 4.0$ for a period of $\sim 0.5 \tau_{\sigma}$ during which the energy ratio peaks at $E_{\text {mag }} / E_{\text {kin }} \approx 0.04$ (labeled "NL" in Fig. 77). A transition occurs to a metastable state $\mathrm{D}^{\prime}$ which has a lifetime of about $2 \tau_{\sigma} \approx 90 \tau_{\nu}$. The MHD system no longer sustains the dynamo process, as the exponential decay of the magnetic field at rate $\gamma_{\mathrm{D}^{\prime}} \approx-0.65$ indicates, until it recovers back to the initial state $\mathrm{D}$ which again allows for magnetic field growth at $\gamma_{\mathrm{D}}$, thus closing the cycle. In the following and unless stated otherwise, the labels D and $\mathrm{D}^{\prime}$ refer to the growth and decay phases as indicated in Fig. 7 .

To investigate the nature of the cyclic behaviour, we have repeated the numerical experiments (ii)-(iv) using flows during both the growth phase $\mathrm{D}$ and the decay phase $\mathrm{D}^{\prime}$. The mean flows and field snapshots were obtained from run (i) at times sufficiently far away from the transitions and while the magnetic field was negligibly small. Experiment (ii) shows growing and decaying solutions for the magnetic field in both cases, $\mathrm{D}$ and $\mathrm{D}^{\prime}$. This result does not disagree with the result obtained from flow $\mathrm{C}$ at $\mathrm{Rm}=44$ since a similar situation can easily be constructed using $\mathrm{D}$ by decreasing $\mathrm{Rm}$ below 44, i.e. all selected snapshots in experiment (ii) are not dynamos whereas a random seed magnetic field grows in time. The 


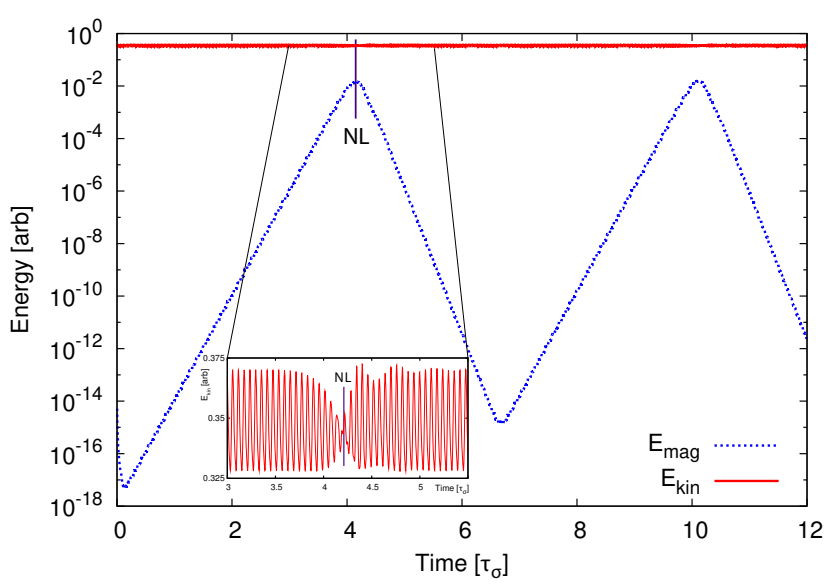

FIG. 7: Time traces of kinetic and magnetic energy, the flow initially being in state $\mathrm{D}(\mathrm{Rm}=44)$. The inset shows the kinetic energy during the transition from growth to decay.

mean-flows during phase $\mathrm{D}$ and $\mathrm{D}^{\prime}$ are identical by visual inspection, which explains the finding that experiment (iii) yields identical decay rates $\gamma_{\mathrm{DM}} \approx-0.31$. Note that during the phase $\mathrm{D}^{\prime}$ in run (i), the magnetic field decays twice as fast as it does in the mean flow. Finally, experiment (iv) yields resonance behaviour qualitatively similar to flow C. To shed more light on the nature of the transitions, further investigations are presented in the following. The main question to be adressed is how the velocity field, the magnetic field, or the combination of both is altered during the transitions.

"Self-killing" (though - to our knowledge - no "selfrecovering") nonlinear dynamos have been reported previously [20, 21]. Their mechanism is based on a modification of the flow due to the action of the Lorentz force away from an initial state which has supported magnetic field growth towards a second solution of the nonlinear Navier-Stokes-Eq. (2) which prevents magnetic field growth. During the transition from $\mathrm{D}$ to $\mathrm{D}^{\prime}$ additional exponentially decaying smooth fluid motions on a slow timescale are excited, as the inset in Fig. 7 indicates. However, the magnetic field continues to decay at constant rate $\gamma_{\mathrm{D}^{\prime}}$ while the fluid perturbation is damped to very small amplitude, indicating that this perturbation might be unimportant. This hypothesis will be confirmed by the investigations presented below.

To scrutinize the transition, we have performed the following numerical experiments (a)-(c) using the solutions $\mathbf{v}_{\mathrm{NL}}$ and/or $\mathbf{B}_{\mathrm{NL}}$ of the system at time $\tau_{\sigma} \approx 4.2$ as initial conditions in the MHD equations. The field $\mathbf{B}_{\mathrm{NL}}$ was rescaled to infinitesimal amplitude, comparable to the seed fields used in previous runs. Hence, the structure of the magnetic field is preserved whereas an immediate backreaction on the flow is avoided. The experiments and results are as follows. (a) Integration of the MHD equations using $\mathbf{v}_{\mathrm{NL}}$ and the rescaled $\mathbf{B}_{\mathrm{NL}}$ as initial conditions exhibits the decay phase at rate $\gamma_{\mathrm{D}^{\prime}}$ [which is of the same duration as in (i)], followed by the transition to growth. (b) Integration starting from $\mathbf{v}_{\mathrm{NL}}$ and an infinitesimal pseudo-random magnetic field yields magnetic field growth at rate $\gamma_{\mathrm{D}}$. (c) Integration starting from a velocity field obtained during $\mathrm{D}^{\prime}$ different from $\mathbf{v}_{\mathrm{NL}}$ and from the rescaled $\mathbf{B}_{\mathrm{NL}}$ (i.e. introducing a phase shift between the self-consistently adjusted configurations of the fields) leads to the same result as in run (b).

We have further investigated the transitions by examining explicitly the temporal characteristics of the spectrum. To this end, Fig. 8 displays time traces of the sum of the volume-integrated poloidal and toroidal energies in the dominant modes during the transition from $\mathrm{D}$ to $\mathrm{D}^{\prime}$. As it was already pointed out in the previous section on hydrodynamics, the $(2,0)$ time trace of flow D is modulated sinusoidally. Hence, it is suited to derive the phase relative to a mode which oscillates similarly in time. In Fig. 8 (a), the dominant modes of kinetic energy obtained from the MHD simulation are plotted over time. These are the $(2,0)$ mode which is responsible for the axisymmetric background flow, and the three strongest $(m=2)$ modes which account for the bulk of the non-axisymmetric wave feature. In addition, the $(2,0)$ time trace from a simulation without magnetic field is shown. The generating run was started using an initial condition obtained from the MHD simulation at $\tau_{\sigma}=2.0$ when the magnetic field was negligibly small. It is seen easily by comparing the $(2,0)$ curves that the nonlinear feedback due to the Lorentz force causes a phase shift by $\pi$ relative to the unperturbed flow. Except for this phase shift, the spectral characteristics of the flow remain unaffected. Fig. 8 (b) shows the dominant magnetic energy modes during the same time interval, and in addition, the time trace of the $(2,0)$ kinetic energy mode from Fig. 8 (a). Before the transition takes place, the oscillations in the dominant modes of the magnetic and the velocity field are in phase, as it is indicated by vertical lines. After the transition, the curves oscillate out of phase by $\pi$. In particular, the magnetic energy modes continue to oscillate at their characteristic frequencies during the transition whereas the kinetic energy modes experience a phase shift by $\pi$. This phase shift is related to a change in the relative alignment of the velocity and magnetic fields which will be discussed in detail in the following paragraph. The relevant spectral components during the transition from $\mathrm{D}^{\prime}$ to $\mathrm{D}$ are displayed in Fig. 9, It is readily seen in subfigure (a) that the velocity field modes are completely unaffected by the change from decay to growth of the magnetic field. The magnetic time traces switch from out-of-phase oscillations to oscillations which are in phase with the dominant hydrodynamic oscillations as subfigure (b) indicates.

It is illustrative to translate the phase shifts to the respective changes in physical space. To this end, we begin with an investigation of the velocity field during the transition NL. The panels (a) and (c) in Fig. 10 show snapshots of the radial component of the velocity field taken at maxima of the $(2,0)$ kinetic energy time trace, 

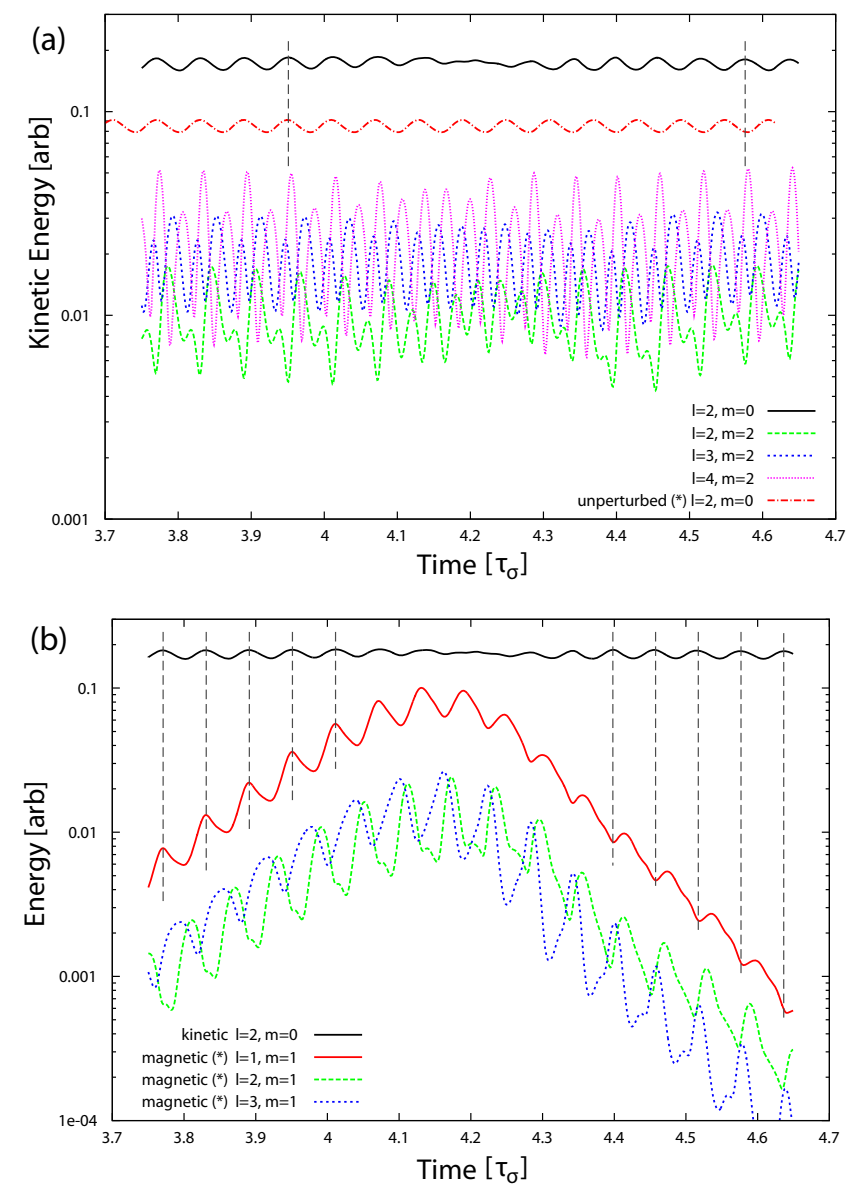

FIG. 8: (a) Volume-integrated spectral energies as functions of time of the 4 dominant modes in flow $\mathrm{D}$ during the transition from $\mathrm{D}$ to $\mathrm{D}^{\prime}$ in the full MHD simulation, c.f. Fig. 7 For comparison, the time trace of the $(2,0)$ mode of a magnetically unperturbed, purely hydrodynamic simulation of the same flow is included, rescaled to $50 \%$ of its amplitude. The scaling is indicated by an asterisk $(*)$. Vertical bars indicate the phase shift which is imposed on the hydrodynamic oscillation. (b) Spectral energies as functions of time of the 3 dominant modes of the magnetic field during the transition from $\mathrm{D}$ to $\mathrm{D}^{\prime}$. For comparison, the $(2,0)$ mode of the velocity field is displayed, cf. subfigure (a). Vertical bars illustrate a phase shift which develops between the oscillations of the magnetic energy and the oscillations of the kinetic energy. The magnetic energy $(*)$ was scaled by a factor of 10 .

before and after the transition, viz. Fig. 8 (a). For the following discussion, it is useful to introduce a virtual plane on a circle of longitude which is defined by the z-axis and the longitudinal coordinate at which the counterpropagating wave features in the upper and lower hemispheres "meet." The position of the plane varies between runs due to the rotational symmetry the flow has about the z-axis before it becomes hydrodynamically unstable. However, once states like C or D are reached, this plane stays fixed in space since the waves counter-propagate at the same velocity in each hemisphere. Relating this virtual plane to the oscillations in Fig. 8 (a), the wave fea-
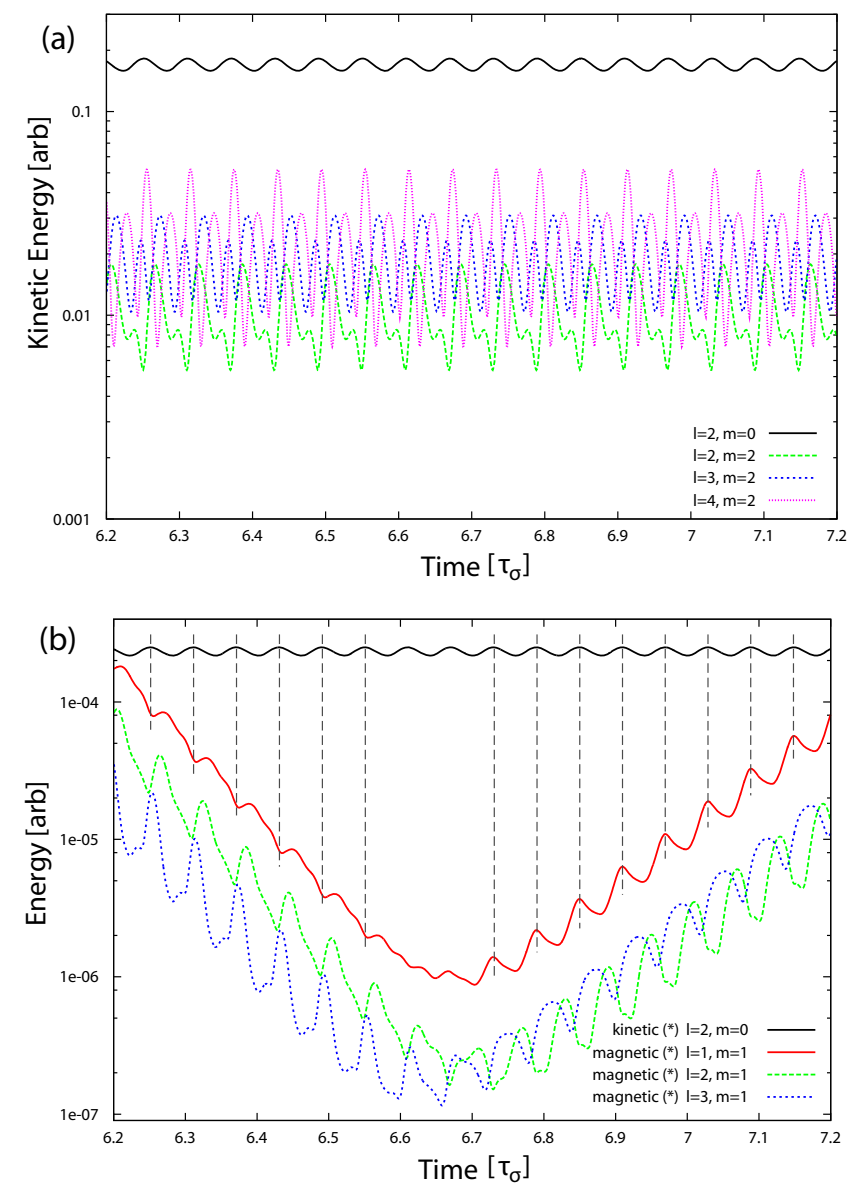

FIG. 9: (a) Dominant modes of the velocity field during the transition from $\mathrm{D}^{\prime}$ to D, viz. Fig. 8 (a). (b) Dominant modes of the magnetic field during the transition from $\mathrm{D}^{\prime}$ to $\mathrm{D}$, viz. Fig. 8 (b). The $(2,0)$ velocity mode from subfigure (a) is displayed for the purpose of illustrating the phase angle relative to the magnetic dipole mode. For practical reasons, the amplitude of the kinetic (magnetic) energy was scaled by a factor of $9^{-3}\left(10^{10}\right)$.

tures reach it at maxima of the $(2,0)$ kinetic energy time trace. From Figs. 10 (a) and (c) it is evident that the nonlinear feedback during NL due to the Lorentz force rotates the plane by $\pi / 2$ about the $\mathrm{z}$-axis which translates to the phase shift by $\pi$ relative to the unperturbed flow as indicated in Fig. 8 (a). The panels (b) and (d) of Fig. 10 show the radial magnetic field snapshots associated with the velocity fields shown in the panels (a) and (c). The magnetic field is dominated by an $(1,1)$ equatorial dipole mode which is evident from the flux patches peaking on the equator. The magnetic field's geometry changes periodically in time during wave motion, however, the equatorial dipole stays fixed sufficiently far from the transitions which can be understood from the same symmetry argument as in the case of the aforementioned plane. The magnetic equatorial dipole does not change its position during NL as a comparison of the panels (b) and (d) in Fig. 10 yields. Comparing the panels (a) and 

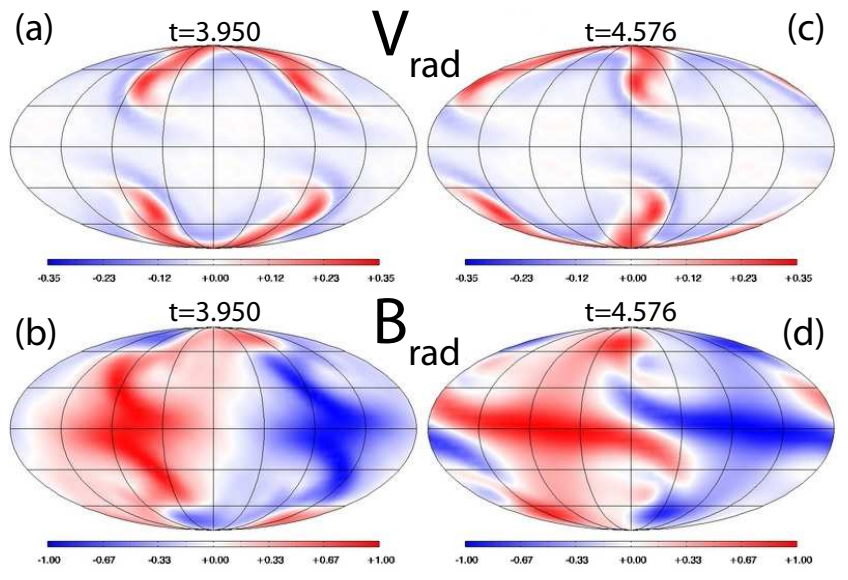

FIG. 10: Radial vector component of the velocity field (a, c) and the normalized magnetic field (b, d) at $90 \%$ of the sphere's radius before $(\mathrm{a}, \mathrm{b})$ and after $(\mathrm{c}, \mathrm{d})$ the first transition from growth to decay, labeled NL. The snapshots were taken at maxima of the $(2,0)$ kinetic energy time trace, viz. Fig. 8 (b).
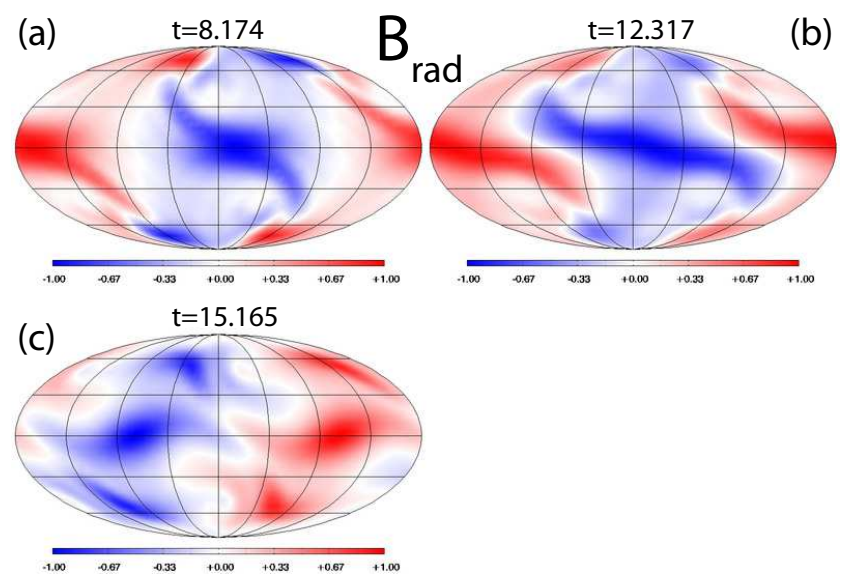

FIG. 11: Radial vector component of the normalized magnetic field at $90 \%$ of the sphere's radius during the second growth phase (a), the second decay phase (b), and the third growth phase (c).

(b) in Fig. 10, the plane characterizing the wave motion is co-aligned with the equatorial dipole before the transition. After NL, the plane and the dipole are oriented perpendicular to each other due to the changes in the velocity field, viz. Fig. 10 (c) and (d). Obviously, the alignment of the velocity field relative to the magnetic field is decisive if the magnetic field grows or decays in time. Let us continue with following the changes to the fields during subsequent transitions. Fig. 11] shows snapshots of the normalized radial magnetic fields during the second growth phase, the second decay phase, and the third growth phase. During the transition from decay to growth at $\tau_{\sigma} \approx 6.7$, the equatorial magnetic dipole rotates by $\pi / 2$ as it is evident from comparing Figs. 10 (d) and 11 (a), whereas the flow remains in the state indi- cated by Figs. 10 (c) and 9 (a). Hence, the magnetic dipole component and the plane characterizing the wave motion are parallel to each other again which results in magnetic field growth. Once the magnetic field has grown sufficiently strong which is the case at $\tau_{\sigma} \approx 10.1$ the wave motion is pushed towards the initial state as depicted in Fig. 10 (a) while the equatorial dipole keeps its position, cf. Fig.111(b). The magnetic field decays and starts growing again when its equatorial dipole component rotates by $\pi / 2$ to reach co-alignment with the plane characterizing the wave motion, cf. Figs. 10 (a) and 11 (c). In summary, the equatorial dipole tilts by $\pi / 2$ about the z-axis between subsequent growth phases which adds up to a field reversal after two cycles, cf. Figs. 10 (b) and 11 (c). The magnetic induction Eq.11allows for such field reversals since if $\mathbf{B}$ is a solution for a given flow, $-\mathbf{B}$ is a valid solution as well. Whenever the magnetic field reacts back on the velocity field, the flow is forced to switch between the configurations shown in Figs. 10 (a) and (c). The dynamo based on flow $\mathrm{C}$ operates similarly to flow $\mathrm{D}$ during the growth phase as it is shown in Figs. 10 (a) and (b), however, does not show cyclic behaviour.

The power spectrum of the growing magnetic field in system D is given in Fig. 12, The power spectrum of the decaying magnetic field is virtually undistinguishable and was therefore not included. Compared to $\mathrm{C}$, the odd $m$ modes are more pronounced which is due to the preference for even $m$ modes in flow D.

Nonlinear saturation to a stationary state with $E_{\text {mag }} / E_{\text {kin }} \approx 1.2$ is reached above $\mathrm{Rm}=51$. Time traces of the dominant kinetic energy modes during saturation are shown in Fig. 13. Compared to the previously discussed cyclic dynamo the system saturates towards a completely stationary state without any oscillations similar to the saturated dynamo state of flow $\mathrm{C}$ at $\mathrm{Rm}=44$. We now turn to a discussion of the physics results.

\section{DISCUSSION}

Our numerical investigations indicate that magnetic field amplification generated by time periodic nonaxisymmetric $m=2$ hydrodynamic waves is relevant to the large-scale dynamo process inside a spherical fluid conductor. These waves emerge due to a hydrodynamic instability which breaks the rotational symmetry of the flow. The flow itself is driven by an axisymmetric body force and subject to the zero-slip outer boundary condition in order to mimic a situation similar to the Madison Dynamo Experiment. Several bifurcation sequences towards different statistically stationary flows with $m=2$ waves are observed depending on the fluid Reynolds number. The numerical investigations are performed in the intermediate regime between the laminar flow and developed turbulence which facilitates the identification of the dominant physical effects.

Let us briefly reiterate the key results which were obtained by solving the induction equation using the afore- 

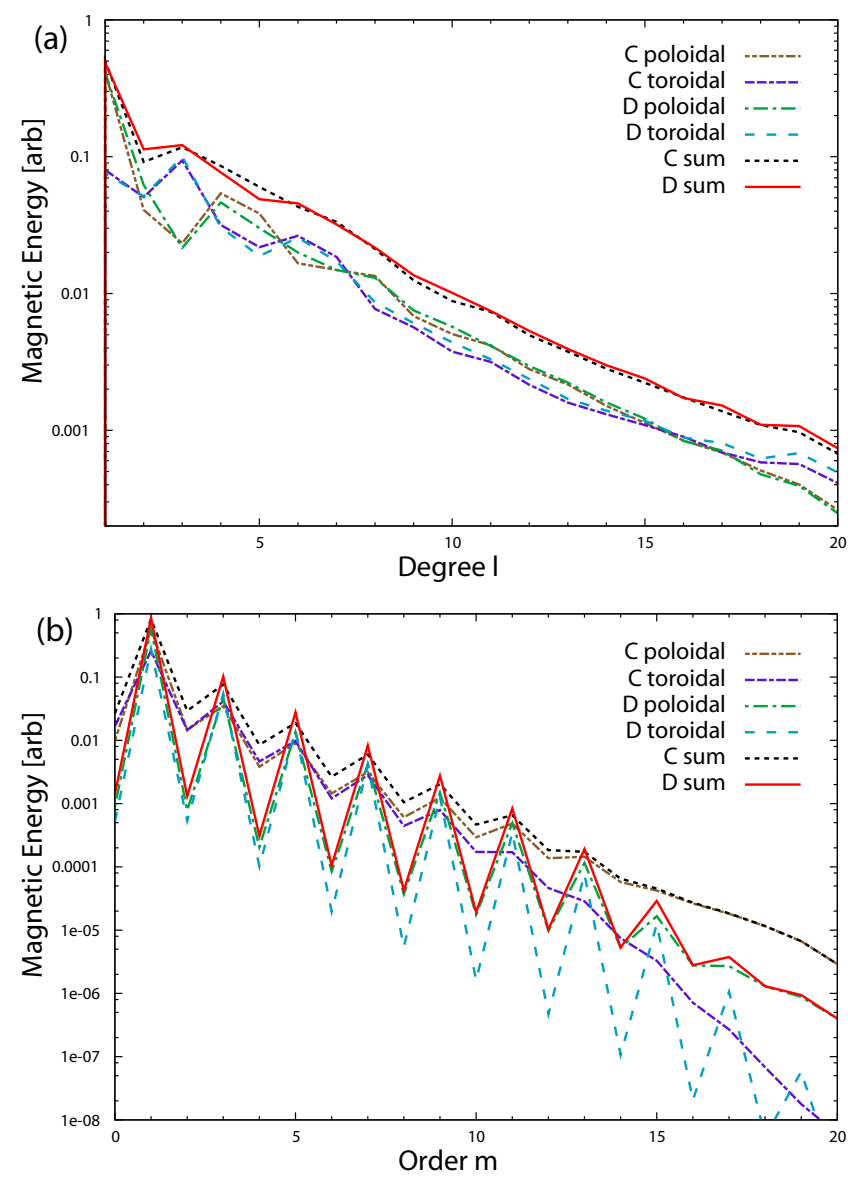

FIG. 12: Normalized and time-averaged spectra of the magnetic energy density during the kinematic (growth) phase in the flows $\mathrm{C}$ and $\mathrm{D}$ as functions of spherical harmonic degree $\ell$ (a) and order $m(\mathrm{~b})$.

mentioned sub-turbulent flows. At a given magnetic Reynolds number, the time-averaged flows as well as snapshots of the flows are not dynamos whereas the timedependent flows with wave motion do show dynamo action. By variation of the wave frequency, maxima in the efficiency of the dynamo process are identified, as well as the breakdown of the dynamo for frequencies which are too low or too high. Moreover, one of the several possible statistically stationary states of the flow shows cyclic dynamo action. We find that the transitions between magnetic field growth and decay crucially depend on the phase angle between characteristic oscillations in the velocity and magnetic energy modes which translates to changes in the relative alignment of the fields in physical space. Hence, system D is different from the self-killing dynamos which have been found previously [20, 21].

These phenomena are symptomatic of dynamo action based on non-normal growth [16]. Non-normal growth refers to perpetual growth due to the mixing of nonorthogonal eigenstates of the time-dependent linear operator $\mathcal{L}(t)$ of the induction equation, which can formally be written as $\partial \mathbf{B} / \partial t=\mathcal{L}(t) \mathbf{B}$. If the time dependence in
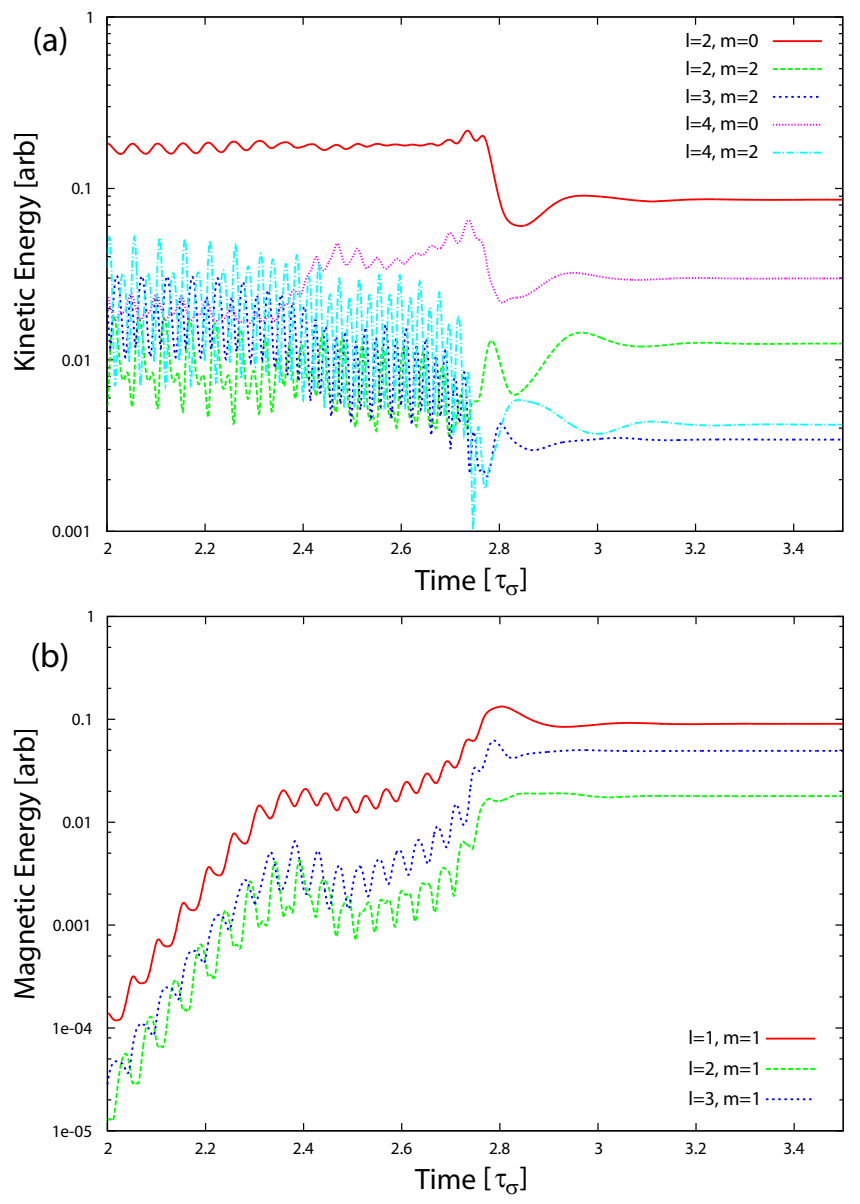

FIG. 13: Saturation of a dynamo based on flow D at $\mathrm{Rm}=51$. The system evolves towards a completely stationary state as it is illustrated by time traces of the dominant kinetic (a) and magnetic (b) modes.

$\mathcal{L}(t)$ given by a non-stationary flow is adequate, perpetual growth of magnetic field modes is possible even if the magnetic field would decay in the absence of time dependence. Such mixing of non-orthogonal eigenstates was shown to act as an efficient driver for dynamo action in Ref. [16], thereby identifying a novel dynamo mechanism different from Lagrangian chaos or the $\alpha$-effect. Using an infinite 2D periodic drifting velocity field which is no dynamo without drift at a given $\mathrm{Rm}$, it was demonstrated that the velocity field supports magnetic field growth for drift velocities not being too fast or too slow. The analogy between our results and the results presented in Ref. 16] strongly supports the conjecture that nonnormal growth is responsible for the magnetic field generation in the $s 2 t 2$ flow with wave motion. Interestingly, flow $\mathrm{C}$ exhibits a wave frequency close to a maximum in dynamo efficiency, cf. Fig. 6. This happens by chance since the wave feature is a hydrodynamic phenomenon and, hence, is independent of a weak seed magnetic field.

The observed saturation of the $s 2 t 2$ dynamo to a state without wave motion confirms the relevance of the ad- 
justment of the wave frequency during the saturation process [16], cf. Fig. 13.

Increasing the Reynolds number above 125 leads to a superposition and interaction of the $m=2$ waves with structures at larger wavenumbers. Turbulence develops which manifests itself in an increase of the critical magnetic Reynolds number $\mathrm{Rm}_{\mathrm{c}}(\mathrm{Re})$, as it was shown numerically for $s 2 t 2$ systems [13, 14, 15]. This increase in the stability curve due to turbulence is a big challenge for laboratory dynamo experiments which use simply connected flows $[3,4,5, \underline{5}, 6$,, 8$]$. Liquid sodium which is preferred as a working fluid has a magnetic Prandtl number $\mathrm{Pm}=\mathrm{Rm} / \mathrm{Re}$ of the order of $10^{-5}$ [19]. Hence, in order to achieve magnetic Reynolds numbers sufficiently large for self-excitation, the experimental flows are vigorously turbulent with Reynolds numbers Re $>10^{5}$, inaccessible to any numerical simulation. Therefore, only extrapolations of $\mathrm{Rm}_{\mathrm{c}}(\mathrm{Re})$ can be done. In numerical studies of dynamos with $\mathrm{Pm}<1$ conducted in infinite periodic boxes, which are usually computationally less expensive than simulations in spherical geometry but lack realistic boundary conditions, the stability curve $\mathrm{Rm}_{\mathrm{c}}(\mathrm{Re})$ was shown to exhibit a saturation to a plateau after an initial increase for large-scale dynamos [9, 10, 11, 12], and similarly for the fundamentally different small-scale dynamo driven by non-helical random forcing, cf. [22] and the references therein. In spherically bounded large-scale dynamos like the system we are focusing on in the present study, the question if and at which level the dynamo threshold saturates to a plateau at large Re has yet to be investigated. Our study shows that field generation due to wave motion is important in the context of the dynamo instability. Beneficial effects are shown to exist in the sub-turbulent regime at low Reynolds numbers of about 100 . The question most interesting to experimenters is, of course, if these results can be transferred to laboratory dynamos. Simulation results obtained for Reynolds numbers above 1000 are encouraging since we still find a pronounced peak at $m=2$ in the spectra of turbulent velocity fields. This indicates that remnants of the $m=2$ waves could be present in the form of coherent structures in experimental flows and potentially act as a driver for magnetic field growth. A modification one might suggest in order to exploit the findings of this paper to improve $s 2 t 2$ setups is the installation of deflector baffles in front of the impellers, designed to force flow components with $m=2$ symmetry on top of the axisymmetric background flow. These baffles could then be rotated about the impeller axis at variable frequency which would allow to search for an optimum in frequency similar to the study presented in Fig. 6.

Growing and transiently decaying magnetic solutions are supported by the flow $\mathrm{D}$. The question if the magnetic field grows is related to an alignment problem of the velocity and magnetic fields. The configuration of $\mathbf{v}$ and $\mathbf{B}$ depicted in Figs. 10 (c) and (d) constitutes a poor state vector of the system since subsequently applying the linear operator $\mathcal{L}(t)$ of the induction equation on $\mathbf{B}$ in order to evolve the system forward in time - which is done by the numerical code-causes the magnetic field to decay. Note that the time-dependence of $\mathcal{L}(t)$ is given by the temporal evolution of the velocity field starting from the configuration shown in Fig. 10 (c). The modification of the flow during the transition from growth to decay is certainly caused by the Lorentz force, however, the reason for the rotation of the magnetic dipole during the transition from decay to growth is not obvious. Slow diffusion of the magnetic field into the preferred position which is then amplified to finally take over is a possible explanation.

Let us finally discuss the relation of the cyclic dynamo $\mathrm{D}$ to the competing axial and transverse dipole modes which are reported for an $s 2 t 2$ flow in [14]. Ref. [14] describes the generation of an axisymmetric magnetic field component via non-axisymmetric turbulent fluctuations in the presence of an axisymmetric $s 2 t 2$ background flow. It is shown that, first, an equatorial dipole magnetic field is generated which creates via Lorentz braking an $m=2$ velocity field component which in turn generates the $m=0$ magnetic field. In the following, the $m=0$ and $m=1$ magnetic modes compete via a modulation of the amplitude of the $m=2$ velocity mode. However, these competing modes are fundamentally different from the cyclic dynamo D which is presented in the paper at hand. First, the $m=2$ non-axisymmetric hydrodynamic waves in our model emerge independently of a magnetic field. Second, when a weak seed magnetic field is introduced, the flows with $m=2$ wave motion generate a dominant $m=1$ transverse dipole component. The axisymmetric components of the magnetic field are relatively weak as the power spectrum in Fig. 12 (b) indicates. Third, there are no competing modes observed. The relative alignment of the magnetic and velocity fields is decisive if the magnetic field grows or decays. In summary, our results are by no means contradictory to the results reported in [14]. Differences in the forcing scheme and in the turbulence intensity exist which are crucial if an axisymmetric dipole is generated. This is pointed out in section III.

In conclusion, magnetic field generation due to wave motion was shown to exist in a spherical $s 2 t 2$ dynamo model. The transfer of these results to experimental flows is not straightforward due to turbulence, nevertheless wave effects could play a role in laboratory dynamos and should be kept in mind when modifying existing or designing new experiments.

KR would like to thank C. C. Finlay and E. J. Spence for helpful conversations. The simulations were performed on the TOK and BOB clusters hosted at RZG. The visualizations in Fig. 2] were created using the VAPOR visualization \& analysis platform [23]. 
[1] H. K. Moffatt, Magnetic field generation in electrically conducting fluids (Cambridge University Press, Cambridge, 1978).

[2] G. Rüdiger and R. Hollerbach, The Magnetic Universe (Wiley-VCH, Weinheim, 2004).

[3] M. D. Nornberg, E. J. Spence, R. D. Kendrick, C. M. Jacobson, and C. B. Forest, Phys. Rev. Lett. 97, 044503 (2006).

[4] E. J. Spence, M. D. Nornberg, C. M. Jacobson, R. D. Kendrick, and C. B. Forest, Phys. Rev. Lett. 96, 055002 (2006).

[5] E. J. Spence, M. D. Nornberg, C. M. Jacobson, C. A. Parada, N. Z. Taylor, R. D. Kendrick, and C. B. Forest, Phys. Rev. Lett. 98, 164503 (2007).

[6] R. Monchaux, M. Berhanu, M. Bourgoin, M. Moulin, P. Odier, J.-F. Pinton, R. Volk, S. Fauve, N. Mordant, F. Pétrélis, et al., Phys. Rev. Lett. 98, 044502 (2007).

[7] F. Ravelet, M. Berhanu, R. Monchaux, S. Aumaître, A. Chiffaudel, F. Daviaud, B. Dubrulle, M. Bourgoin, P. Odier, N. Plihon, et al., Phys. Rev. Lett. 101, 074502 (2008).

[8] F. Stefani, A. Gailitis, and G. Gerbeth, ZAMM 88, 930 (2008).

[9] Y. Ponty, P. D. Mininni, D. C. Montgomery, J.-F. Pinton, H. Politano, and A. Pouquet, Phys. Rev. Lett. 94, 164502 (2005).

[10] P. D. Mininni, Phys. Plasmas 13, 056502 (2006).

[11] J.-P. Laval, P. Blaineau, N. Leprovost, B. Dubrulle, and
F. Daviaud, Phys. Rev. Lett. 96, 204503 (2006).

[12] Y. Ponty, P. D. Mininni, J.-F. Pinton, H. Politano, and A. Pouquet, New J. Phys. 9, 296 (2007).

[13] R. A. Bayliss, C. B. Forest, M. D. Nornberg, E. J. Spence, and P. W. Terry, Phys. Rev. E 75, 026303 (2007).

[14] C. Gissinger, E. Dormy, and S. Fauve, Phys. Rev. Lett. 101, 144502 (2008).

[15] K. Reuter, F. Jenko, and C. B. Forest, New J. Phys. 11, 013027 (2009).

[16] A. Tilgner, Phys. Rev. Lett. 100, 128501 (2008).

[17] K. Reuter, F. Jenko, C. B. Forest, and A. R. Bayliss, Comput. Phys. Commun. 179, 245 (2008).

[18] M. L. Dudley and R. W. James, Proc. R. Soc. Lond. A 425, 407 (1989).

[19] M. D. Nornberg, E. J. Spence, R. D. Kendrick, C. M. Jacobson, and C. B. Forest, Phys. Plasmas 13, 055901 (2006).

[20] N. H. Brummell, F. Cattaneo, and S. M. Tobias, Physics Letters A 249, 437 (1998).

[21] H. Fuchs, K.-H. Rädler, and M. Rheinhard, Astron. Nachr. 320, 129 (1999).

[22] A. A. Schekochihin, A. B. Iskakov, S. C. Cowley, J. C. McWilliams, M. R. E. Proctor, and T. A. Yousef, New J. Phys. 9, 300 (2007).

[23] J. Clyne, P. Mininni, A. Norton, and M. Rast, New J. Phys. 9, 301 (2007). 